\title{
Improvements in malaria testing and treatment after a national community health worker program in rural Liberia
}

\author{
Jeffrey W. Rozelle ${ }^{1} \odot$, Jerome Korvah², Olasford Wiah², John Kraemer $^{3} \odot$, Lisa R. Hirschhorn ${ }^{4} \odot$, Matt R. Price ${ }^{5} \oplus$ \\ Marion Subah', Lisha McCormick', Brittney Varpilah', Rajesh Panjabi ${ }^{6}$ \\ ${ }^{1}$ Last Mile Health, Monrovia, Liberia, ${ }^{2}$ Ministry of Health, Liberia, Monrovia, Liberia, ${ }^{3}$ Last Mile Health, Monrovia, Liberia; Georgetown University, \\ Washington DC, United States, ${ }^{4}$ Last Mile Health, Boston, Massachusetts, United States; Northwestern University, Chicago, Illinois, United States, 5 \\ Last Mile Health, Boston, Massachusetts, United States, ${ }^{6}$ Last Mile Health, Boston, Massachusetts, United States; Harvard Medical School, Boston, \\ Massachusetts, United States \\ Keywords: primary health care, covid-19, community health workers, malaria \\ https://doi.org/10.29392/001c.25979
}

\section{Journal of Global Health Reports}

Vol. 5, 2021

\begin{abstract}
Background
Progress in reducing malaria incidence and deaths has stalled, in part due to limited access to quality malaria testing and treatment amongst rural populations. This time-series analysis aims to describe changes in rural malaria diagnosis and treatment before and during the rollout of Liberia's National Community Health Assistant (CHA) program. It also explores how malaria service delivery changed during the COVID-19 epidemic.
\end{abstract}

\begin{abstract}
Methods
Malaria diagnosis and treatment data from 315 rural health facility catchments supported by Liberia's National CHA Program were collected from Liberia's Health Management Information System and analyzed. Trends in malaria service delivery coverage and quality were assessed between January 2011 and December 2020.
\end{abstract}

\section{Results}

By 2020, four years after the program's introduction, Liberia's Community Health Assistants diagnosed $50 \%$ of rapid diagnostic test (RDT) or microscopy-confirmed malaria cases and carried out $51 \%$ of malaria treatments amongst children under age five in rural areas where CHAs were present. Furthermore, the percentage of malaria diagnoses that were confirmed by microscopy or RDT increased from $71 \%$ prior to the program to $95 \%$ in 2020. These results were effectively sustained in rural and remote communities during COVID-19 in 2020

\section{Conclusions}

The introduction of Liberia's CHA program was associated with improvements in the quality of malaria diagnoses and contributed to treating a significant share of the malaria burden amongst children under age five in remote rural communities across Liberia, and these improvements were sustained during COVID-19. Investments made in rural community health systems can be leveraged by policy makers to strengthen malaria diagnosis, treatment and control, especially in the age of COVID-19.

The 2020 World Malaria Report observes that progress has stalled in reducing malaria incidence and deaths across many countries. ${ }^{1}$ Global malaria case incidence declined by $27 \%$ between 2000 and 2015, and by less than $2 \%$ between 2015 and 2019, indicating a slowing of the rate of decline since 2015. Globally, malaria deaths per 100000 population at risk reduced from about 25 in 2000 to 12 in 2015 and 10 in 2019 , with the rate of decline slowing in recent years. ${ }^{1}$ The COVID-19 pandemic threatened to further slow progress against malaria, with the World Health Organization (WHO) reporting malaria diagnosis and treatment moderately or severely disrupted in $44 \%$ of countries.
An important barrier to global malaria control is the lack of access to quality testing, and in 2019 the WHO reported that $31 \%$ of people with fever sub-Saharan Africa did not seek treatment, a figure worse in rural areas, including in high malaria prevalence zones. ${ }^{1}$ Rural children in Liberia have a high prevalence of malaria, with nearly two thirds (62\%) aged 6-59 months testing positive in Liberia's 2016 Malaria Indicator Survey - more than double the prevalence of their urban counterparts. ${ }^{2}$ However, care-seeking for children with fever in Liberia's 2019-20 Demographic and Health Survey was lower in rural (74\%) compared to urban settings (87\%) in 2019, and the quality of care is low with 
only one-half (51\%) of rural children with fever tested for malaria. ${ }^{3}$ While integrated management of children illness (IMCI) is a widely adopted evidence-based strategy to improve testing and treatment for children with malaria, work is needed to continue to close access and quality gaps in malaria diagnosis and treatment particularly for rural children for whom access to facilities is challenging. ${ }^{4-6}$

Following the 2014-16 West Africa Ebola epidemic, the Government of Liberia revised its 2008 Community Health Services Policy with the aim expanding essential health services, including malaria testing and treatment, in underserved remote communities, defined as those more than $5 \mathrm{~km}$ from a health facility. ${ }^{7}$ Liberia's National Community Health Assistant (CHA) program, launched in July 2016, paid community health workers (instead of keeping them as volunteers), increased their density in remote communities (where their numbers had been low and case loads were high), trained and supervised them with nurses and other frontline clinicians (when previously they were supervised by non-clinicians), and equipped them with rapid diagnostic tests (RDTs) and artemisinin combination treatments (ACTs), along with medicines and supplies to deliver other essential health services like contraceptives, antibiotics for acute respiratory infection (which they had not been supplied with previously). Leadership, collaboration, and resources from Liberia's Ministry of Health, the U.S. President's Malaria Initiative, Global Fund, World Bank, Global Financing Facility, and non-governmental and philanthropic partners enabled rapid scaling of malaria diagnosis and treatment and other essential health services through this new national platform. By December 2020, 3574 CHAs and 373 supervisors were serving people in remote catchment communities linked with 315 government clinics reporting data.

This study describes changes in malaria diagnosis and treatment in the public sector in the setting of the Liberia's nationally scaled CHA program. In particular, it aims to describe changes in rural malaria diagnosis and treatment before and during national community health worker program scale-up. Further, it aims to examine whether, and if so, how malaria service delivery changed during the 2020 COVID-19 epidemic, as a test of the resilience of rural health systems.

\section{METHODS}

\section{STUDY SETTING}

Launched in 2016, Liberia's national policy recommends that a maximum of ten CHAs should be supervised by one nurse or other clinician (e.g. physician assistant) based at clinics affiliated with the CHA's catchment population of up to 350 people. As previously described, ${ }^{8}$ the CHA program introduced a package of preventive and curative services delivered by CHAs, including community-event based surveillance for infectious disease events, supportive care for patients with HIV and tuberculosis, and reproductive, maternal, newborn and child health services, among others. Child health services focused on introducing integrated community case management of illnesses for children under age five, including treatment for diarrhea (with oral re- hydration solution (ORS) and zinc), acute respiratory infection (with amoxicillin), screening for severe and moderate acute malnutrition, and malaria testing (with Rapid Diagnostic Test (RDTs) and treatment with Artemisinin-based Combination Therapy (ACT) for uncomplicated malaria. In cases of RDT/ACT stockout or complicated malaria, CHAs were trained to refer children to health facilities. Facility malaria diagnosis occurred via RDT, microscopy, or clinical diagnosis when diagnostic tests were unavailable or clinicians doubted diagnostic results, with treatment of uncomplicated malaria occurring via ACTs.

In response to COVID-19, Liberia's Ministry of Health updated the CHA program's protocols for malaria service delivery to prioritize safety of health workers and patients. CHAs were provided face masks and gloves. When a child under age five presented with a fever, CHAs were trained to screen each patient and their household contacts for symptoms of COVID-19. If a trigger was identified, CHAs would counsel the patient on how to safely self-isolate and contact the surveillance team for further investigation. Patients with suspected COVID-19 were referred to the health facility for further assessment, and those not meeting the case definition were tested for malaria and treated according to protocols. $^{9}$

\section{DATA SOURCES}

We restricted analyses to data collected through the 315 Liberian health facilities with available National CHA Program community-level data in December 2020. Included data are from both the national community-based data systems and facility-based data systems, which are reported through facilities. All monthly reports of our outcomes of interest from 2011 to 2020 were extracted from these 315 health facilities from the DHIS 2 using the httr and jsonlite $\mathrm{R}$ packages, regardless of when the CHA program started at that facility.

Records of clinical encounters with CHAs are captured in Liberia's paper-based Community Based Information System (CBIS). CHAs' supervisors collect and aggregate reports of services delivered on a monthly basis and verify that counts match service delivery records. On a monthly basis, aggregated counts are entered into the District Health Information System (DHIS2) platform. Similarly, health facilities maintain paper records of clinical encounters, and services delivered. On a monthly basis, health facilities complete an aggregated report, which is then collected collected by the County Health Team and entered in to the DHIS2 platform online.

\section{DATA ANALYSIS}

Outcomes of interest include: (i) malaria diagnosis episodes by year, disaggregated by provider type (facility vs CHW) and diagnosis type (clinical, RDT, and microscopy), and (ii) malaria treatment episodes by month and year, disaggregated by provider type (facility vs $\mathrm{CHW}$ ). Provider and patient gender were not collected across the entire study period and are therefore are not reported. With longitudinal data from 2011-2020, we examined data from three periods: (i) before the 2017 introduction of Liberia's National CHA 
Program, (ii) during the 2017-2020 scale-up of Liberia's National CHA Program, and (iii) following the first reported COVID-19 case in March 2020. We conducted a descriptive analysis of the service delivery outputs of health facilities and community health workers over time and presented these in charts for ease of interpretation.

Finally, at the recommendation of a reviewer, we included a post-hoc additive Holt-Winter model to better visualize changes over time and assess whether total number of treatment episodes deviated from previous trends during the COVID-19 period while accounting for seasonality (Figure S1 in the Online Supplementary Document). The model was fitted using the base $\mathrm{R}$ HoltWinters function on data from January 2016 until March 2020. From there, the forecast was extracted from the Holt-Winters model using the predict function for months through the end of 2020 and visually compared with the observed number of treatment episodes.

\section{RESULTS}

The first CHA-conducted malaria diagnoses began in 2017, when the national program was introduced and began collecting data from 12 of Liberia's 15 counties by December 2017 (Table S1 in the Online Supplementary Document). The introduction of Liberia's CHA Program was associated with a shift in malaria diagnostic methods in the CHA-supported catchment areas (Figure 1) amongst children under age five. In 2016, prior to the introduction of the CHA program, only $40 \%$ of reported diagnoses were confirmed using RDT or microscopy. In 2017-2018 (first full implementation year), CHAs provided 133,002 diagnoses, and this increased to 146,319 in 2020 as the program expanded (Figure 1). In 2020 , $48 \%$ of all malaria diagnoses in the 315 CHA-supported catchment areas were made by CHAs (Figure 1). During the CHA program period, there was a consistent trend toward diagnosis by CHAs rather than at health facilities, suggesting that community care was substituted for facility-based care.

By 2020, four years following rollout of the CHA program, the percentage of malaria diagnoses that were confirmed by microscopy or RDT increased from $71 \%$ in 2016 to $95 \%$ in 2020. Among confirmed diagnoses, 50\% were conducted by CHAs.

Across the 315 health facilities included in the study, the number of malaria treatment episodes for children under age five (Figure 2) appeared relatively stable between 2011-16, at around 300,000 cases of malaria treated, except during the Ebola outbreak. Annual treatment figures recovered by 2016.

After the introduction of the CHA program in 2017, there was a substantial shift in treatment seeking patterns. The total number of treatment episodes did not change markedly. However, a growing proportion of treatment was provided by CHAs. By 2019, 45\% of treatments provided to children under-age five diagnosed with malaria in included catchment areas were from a CHA.

In 2020, and nine months after the first case of COVID-19 was identified, malaria testing was sustained nationally, with CHAs responsible for $52 \%$ of malaria diagnoses from April - December 2020, compared to 47\% during the same

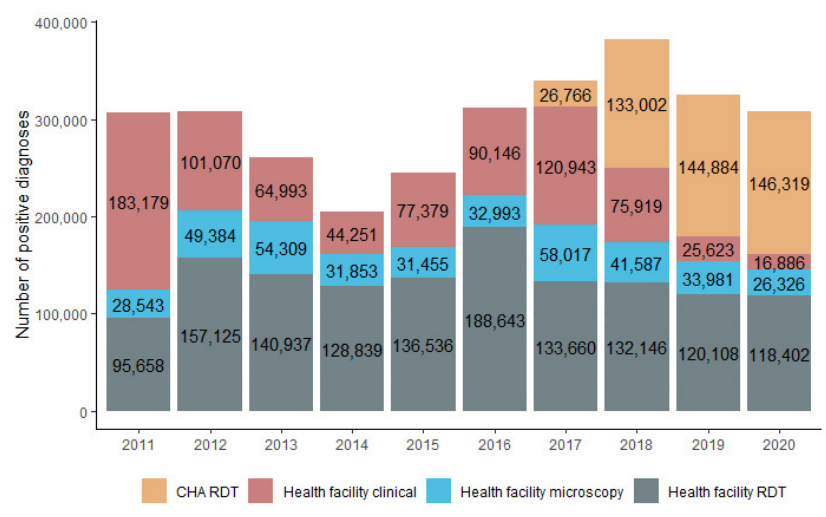

Figure 1. Annual numbers of reported malaria diagnosis by provider and type.

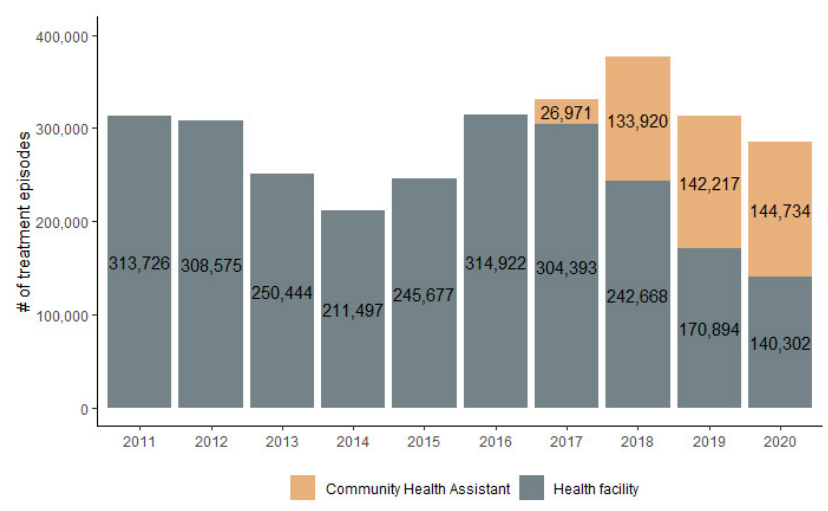

Figure 2. Malaria treatment annually by provider among facilities in the National Community Health Assistant Program, children under five.

months of 2019, in line with the slightly increased scale of the program. Additionally, the proportion of treatments delivered at community level increased to $51 \%$ in 2020 as the National CHA Program continued to scale up, from 3307 CHAs in December 2019 to 3574 CHAs in December 2020.

The number of malaria treatments remained approximately constant over seasons compared to pre-pandemic years (Figure 3). The total number of treatment episodes after the first COVID-19 case in Liberia remained within the 95\% confidence interval of the Additive Holt-Winters forecast using data from the 4 previous years (Figure S1 in the Online Supplementary Document).

\section{DISCUSSION}

Introduction of Liberia's CHA program was associated with improvements in the quality of malaria diagnoses and contributed to treating a significant share of the malaria burden amongst children under age five in remote rural communities across Liberia. In addition, we found that these improvements were sustained during COVID-19. By 2020, more cases were confirmed with microscopy or RDT than in previous years, and CHAs diagnosed nearly half of the test- 
confirmed malaria cases and delivered more than half of the treatments to children under five in rural populations in the study. These improvements in national rural malaria testing and treatment were maintained during the first year of COVID-19 pandemic.

By employing CHAs and increasing their density in remote communities, supervising them with clinicians and creating supply chains, Liberia improved the systems supporting community health workers, not just the services they deliver. In so doing, Liberia was able to decentralize malaria RDT and treatment from the facilities to the home, improving the share of diagnoses that are confirmed and improving treatment for rural children. Findings from our health services research are supported by separate population-level studies including the 2019-20 Demographic and Health Survey in Liberia, which showed the percentage of children with a blood prick for malaria testing was higher for the first time in rural areas than urban areas, indicating greater access to testing in rural areas. ${ }^{3}$

Community-based service delivery brings multiple benefits at patient, population and health system levels. The observation that nearly half of all children under five in these areas received care for malaria from a CHA means more convenient care without user fees for most families. In other studies, at a county level, we have found families report improved access to testing and care-seeking for fever improves and reduced use of unqualified providers. Treatment at home means saving the opportunity cost from losing a day of work to secure treatment for a child and creates savings on transportation. Quality also includes timeliness, another advantage of CHA-delivered care allowing febrile children to receive care more quickly. ${ }^{6}$ Global evidence demonstrates that distance is a critical determinant of timely treatment, and that traveling more than 3-5 kilometers to a health facility for malaria care delays care and careseeking. ${ }^{10-15}$ Treating malaria early in the community directly addresses this barrier, and early treatment reduces negative outcomes of the disease in children. ${ }^{16}$ Additionally, community-based delivery is likely a more cost-effective mechanism compared to clinic delivered care, though the extent of this would be an important investigation for future research.

Increased testing and treatment availability at the community level appears to coincide with relative decreases in utilization of malaria testing and treatment at health facility level. Outpatient Department data from DHIS2 shows that at about half of all causes of Outpatient Department visits by children under age five in these 315 health facilities was for malaria. Decentralizing access to quality malaria care for rural populations by increasing malaria RDT confirmation can reduce misuse of ACTs, a cornerstone of the WHO's Global plan for artemisinin resistance containment. ${ }^{17}$ Our study CHA program has allowed more remote children who previously relied on health facilities to be tested and treated in their homes by CHAs, consistent with studies including randomized controlled trials in Liberia, Mali, and Uganda. 6,18-20

In 2020, the COVID-19 pandemic threatened to disrupt malaria and other primary health care services. Globally, the WHO reported that COVID-19 control measures (e.g. physical distancing, business closures, transportation dis-

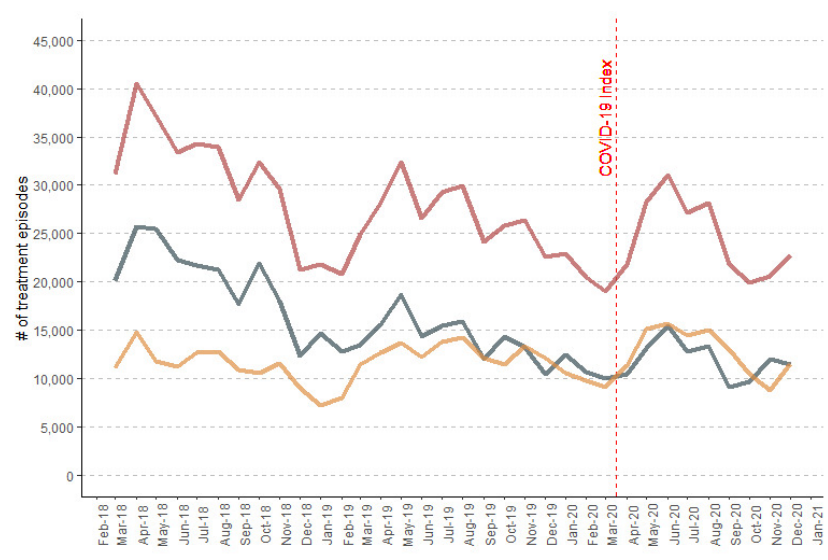

Figure 3. Malaria treatment episodes before and during the COVID-19 pandemic, by provider.

ruptions) and community fear of infection disrupted the access, distribution of essential malaria supplies, including tests and treatments. ${ }^{21}$ Although Liberia has a lower COVID-19 case load compared to some of its peers in the region, facility closures impacted utilization of select facility-based primary healthcare services in a recent analysis. ${ }^{22}$ However, our study found that community-based malaria testing and treatment services for rural children with malaria appear to be maintained in the first year of the COVID-19 pandemic, and without notable decreases in facility-based malaria testing and treatment. Shortly after COVID-19 was detected in Liberia in March 2020, Liberia's Ministry of Health leveraged systems - including supervision, supply chain - supporting CHAs. For instance, they provided personal protective equipment to CHAs and adapted diagnostic and treatment algorithms accordingly. While it's unclear to what extent these changes in protocols were put into practice, PPE uptake was wide, and in Liberia and elsewhere there are reports of community-based provider's decreases in anxiety, fear and improvements in safety to continue providing services like malaria testing and treatment. ${ }^{23}$ The actual number of total treatments delivered also fell within the $95 \%$ CI of projections from a seasonal Holt-Winters model based on the 4 years prior to the first COVID-19 case (Figure S1 in the Online Supplementary Document), though disentangling changes in disease burden from changes in coverage remains a challenge in this model.

There were a number of limitations in our study. The data analyzed is a full census of reported diagnoses and treatment, so there is not sampling error. However, there is a possibility of non-sampling error. DHIS2 and similar data systems have known limitations in interpreting malaria trends and do not capture data from informal, traditional, or private sector providers. ${ }^{24}$ This dataset alone is therefore insufficient to accurately measure national malaria diagnosis and treatment trends or to attribute the continuation of treatment, and episodes treated at both facility and community levels may be double-counted. Some government supported community health volunteers provided diagnosis 
and treatment at smaller scales prior to 2016, but these data were not entered in government databases. Timeliness and correctness of diagnosis and treatment are not directly measured in this study. Furthermore, it is possible that there is differential data quality in data reported by CHAs and by health facilities. Mismatches between malaria treatment counts disaggregated by time and age in $9 \%$ of CHSS reports included in this analysis suggest data completeness, consistency, and quality may vary, but do not appear to alter conclusions. These data are best used to describe and interpret shifting norms in malaria service delivery and quality and could be extended through subnational analyses of variation in quality and performance.

These data have significant policy and practice implications for community health workforce investments, particularly during COVID-19. Despite inclusion of "malaria services" in Liberia 2008 National Community Health Services Policy, Liberia's community health workforce was unpaid and poorly supervised, with high attrition and limited effectiveness. Studies showed very low coverage rates of essential health services during this time, ${ }^{25}$ which limited timely access to malaria testing and treatment. After malaria services collapsed during the Ebola outbreak, Liberia's Ministry of Health and partners made a commitment to change policy. The Health Ministry and partners recognized the need to invest not only in the services community health workers, but in systems to support them. Liberia revised its community health services policy and launched the CHA program. The Health Ministry decided that community health assistants would be selected in remote rural communities, increasing geographic coverage; supervised by nurses and other primary care clinicians; up-skilled including training in malaria testing and treatment as part of a broader set of primary care interventions; supplied with RDTs and ACTs; and provided salary support and a contract. These systems investments are consistent with other exemplar community health programs that have adapted the WHO guidelines on optimizing systems and policy for community health programs - and they can be extended by policymakers seeking to leverage community health systems to strengthen malaria diagnosis, treatment and control, especially in the age of COVID-19. Similar findings in other contexts would be useful to strengthen the generalizability of these findings.

\section{ACKNOWLEDGMENTS}

The authors are grateful to Liberia's Ministry of Health, Liberia's National Malaria Control Program and Community Health Services Division, county health teams, community health workforce, partners, and community members - as well as the U.S. President's Malaria Initiative, USAID, CDC, Global Fund, World Bank, Global Financing Facility, Bill \& Melinda Gates Foundation, Co-Impact, the Audacious Project, World Health Organization, UNICEF, Roll Back Malaria partnership, and philanthropic partners for their investments in malaria control in Liberia. We are grateful to D. Levi Hinneh from Liberia's National Malaria Control Program for valuable comments. Finally, we want to thank CHAs, CHSSs, and frontline workers in Liberia for their commitment to maintaining malaria treatment before and during the COVID-19 pandemic.

\section{FUNDING}

None

\section{AUTHORSHIP CONTRIBUTIONS}

All authors contributed to the draft and final manuscript.

\section{CONFLICTS OF INTERESTS}

The authors completed the Unified Competing Interest form at http://www.icmje.org/disclosure-of-interest/ (available upon request from the corresponding author) and declare no conflicts of interest.

\section{CORRESPONDENCE TO:}

\section{Lisa R Hirschhorn, MD, MPH}

Professor of Medical Social Sciences and Psychiatry and Behavioral Sciences

625 Michigan Ave 14-013, Chicago, IL 60611

Lisa.Hirschhorn@Northwestern.edu

Submitted: February 07, 2021 GMT, Accepted: July 06, 2021

GMT 


\section{REFERENCES}

1. World Health Organization. World Malaria Report.; 2020. https://apps.who.int/iris/rest/bitstreams/13218 72/retrieve

\section{National Malaria Control Program (NMCP)} [Liberia], Ministry of Health (MOH), Liberia Institute of Statistics and Geo-Information Services (LISGIS), ICF. Liberia: Malaria Indicator Survey 2016.; 2017. http s://dhsprogram.com/pubs/pdf/MIS27/MIS27.pdf

3. Liberia Institute of Statistics and Geo-Information Services, Liberian Ministry of Health and Social Welfare, Liberian National AIDS Control Program. Liberia Demographic and Health Survey 2019-20: Preliminary Report.; 2020. https://web.archive.org/we b/20210224185605/dhsprogram.com/pubs/pdf/PR117/ PR117.pdf

4. Christopher JB, Le May A, Lewin S, Ross DA. Thirty years after Alma-Ata: A systematic review of the impact of community health workers delivering curative interventions against malaria, pneumonia and diarrhoea on child mortality and morbidity in sub-Saharan Africa. Hum Resour Health. 2011;9(1):1-11. doi:10.1186/1478-4491-9-27

5. Luckow PW, Kenny A, White E, et al. Implementation research on community health workers' provision of maternal and child health services in rural Liberia. Bull World Health Organ. 2017;95(2):119-120. doi:10.2471/BLT.16.175513

6. White EE, Downey J, Sathananthan V, et al. A community health worker intervention to increase childhood disease treatment coverage in rural Liberia: A controlled before-and-after evaluation. Am J Public Health. 2018;108(9):1252-1259. doi:10.2105/AJPH.201 8.304555

7. Paying and investing in last-mile community health workers accelerates universal health coverage. The BMJ. Published 2018. https://blogs.bmj.cohttps://blog s.bmj.com/bmj/2018/05/22/paying-and-investing-in-1 ast-mile-community-health-workers-accelerates-uni versal-health-coverage/

8. Exemplars in Global Health. Community Health Workers in Liberia. Exemplars in Global Health. Published 2020. Accessed December 31, 2020. http s://www.exemplars.health/topics/community-healthworkers/liberia

9. Liberia Ministry of Health. Community Health Worker Handbook for COVID-19. Published online 2020.
10. Workineh B, Mekonnen FA. Early treatmentseeking behaviour for malaria in febrile patients in northwest Ethiopia. Malar J. 2018;17(1):1-8. doi:10.11 86/s12936-018-2556-2

11. Turuse EA, Gelaye KA, Beyen TK. Determinants of Delay in Malaria Prompt Diagnosis and Timely Treatment among Under-Five Children in Shashogo Woreda, Hadiya Zone, Southern Ethiopia: A Case Control Study. Health. 2014;6(10):950-959. doi:10.423 6/health.2014.610120

12. Getahun A, Deribe K, Deribew A. Determinants of delay in malaria treatment-seeking behaviour for under-five children in south-west Ethiopia: A case control study. Malar J. 2010;9(1):320. doi:10.1186/147 5-2875-9-320

13. Xu JW, Xu QZ, Liu H, Zeng YR. Malaria treatmentseeking behaviour and related factors of Wa ethnic minority in Myanmar: A cross-sectional study. Malar J. 2012;11(1):1-7. doi:10.1186/1475-2875-11-417

14. Das A, Ravindran TS. Factors affecting treatmentseeking for febrile illness in a malaria endemic block in Boudh district, Orissa, India: Policy implications for malaria control. Malar J. 2010;9(1):1-10. doi:10.11 86/1475-2875-9-377

15. Romay-Barja M, Cano J, Ncogo P, et al. Determinants of delay in malaria care-seeking behaviour for children 15 years and under in Bata district, Equatorial Guinea. Malar J. 2016;15(1):187. $\underline{\mathrm{d}}$ oi:10.1186/s12936-016-1239-0

16. WHO. Overview of Malaria Treatment. World Health Organization; 2016. Accessed September 15, 2020. http://www.who.int/malaria/areas/treatment/ov erview/en

\section{WHO. Global Plan for Artemisinin Resistance} Containment.; 2011. Accessed September 2, 2020. htt p://www.who.int/malaria/publications/atoz/artemisin in resistance containment 2011.pdf

18. Johnson AD, Thomson DR, Atwood S, et al. Assessing early access to care and child survival during a health system strengthening intervention in Mali: A repeated cross sectional survey. PLOS ONE. 2013;8(12):e81304. doi:10.1371/journal.pone.008130 $\underline{4}$

19. Parpia AS, Ndeffo-Mbah ML, Wenzel NS, Galvani AP. Effects of response to 2014-2015 ebola outbreak on deaths from malaria, HIV/AIDS, and tuberculosis, West Africa. Emerg Infect Dis. 2016;22(3):433-441. do i:10.3201/eid2203.150977 
20. Nyqvist MB, Guariso A, Svensson J, YanagizawaDrott D. Reducing child mortality in the last mile: Experimental evidence on community health promoters in Uganda. Am Econ J Appl Econ. 2019;11(3):155-192. doi:10.1257/app.20170201

21. WHO Team. The Potential Impact of Health Service Disruptions on the Burden of Malaria.; 2020. Accessed August 26, 2020. https://www.who.int/publications/i/i tem/9789240004641

22. Ahmed T, Fernandez PA, Drouard S, et al. Monitoring continuity of essential health services during the COVID-19 Pandemic. Published 2020. http s://www.globalfinancingfacility.org/monitoring-conti nuity-essential-health-services-during-covid-19-pan $\underline{\text { demic }}$
23. Wiah L, Subah M, Varpilah B, et al. Prevent, detect, respond: How community health workers can help in the fight against covid-19. BMJ London.

Published online 2020:1-4. https://blogs.bmj.com/bm $\mathrm{j} / 2020 / 03 / 27 /$ prevent-detect-respond-how-communit $\mathrm{y}$-health-workers-can-help-fight-covid-19/

24. Rowe AK, Kachur SP, Yoon SS, Lynch M, Slutsker L, Steketee RW. Caution is required when using health facility-based data to evaluate the health impact of malaria control efforts in Africa. Malar J. 2009;8(1):1-3. doi:10.1186/1475-2875-8-209

25. Kentoffio K, Kraemer JD, Griffiths T, et al. Charting health system reconstruction in post-war Liberia: A comparison of rural vs. remote healthcare utilization. BMC Health Serv Res. 2016;16(1):478. do i:10.1186/s12913-016-1709-7 


\section{SUPPLEMENTARY MATERIALS}

\section{Online Supplementary Document}

Download: https://www.joghr.org/article/25979-improvements-in-malaria-testing-and-treatment-after-a-nationalcommunity-health-worker-program-in-rural-liberia/attachment/66295.docx 